\title{
Preliminary indications for antibiotic susceptibility tests in less than six hour in positive blood cultures
}

\author{
Vesselina Kroumova, Elisa Gobbato, Paola Macaluso, Sabrina Tamburelli, Francesca Marini, Marcella Perone, \\ Stefania Orlandi, Morena Viviani, Giacomo Fortina \\ Laboratorio di Microbiologia e Virologia Azienda Ospedaliera Universitaria “Maggiore della Carità”, Novara
}

Key words: Antimicrobial susceptibility tests; Sepsis, Critical illness

Indicazioni preliminari di antibiotico-sensibilità in meno di sei ore in emocolture positive

\section{SUMMARY}

A rapid determination of the antibiotic susceptibility patterns of pathogens responsible for sepsis represents a significant milestone for a timely correct antibiotic therapy. The system HB\&L® (ALIFAX) allows reduced time in the detection of bacterial growth and consequently is able to detect the growth or absence of certain microorganisms in the presence of a given antibiotic. In this study three system for rapid antibiotic susceptibility tests among bacteria isolated from blood were compared: HB\&L ${ }^{\circledR}$ (ALIFAX),VITEK ${ }^{\circledR} 2$ (bioMérieux) and essays Etest ${ }^{\circledR}$ (bioMérieux).

Present findings indicate that $\mathrm{HB} \& \mathrm{~L}^{\circledR}$ (ALIFAX) is rapid reliable instrument that may support the clinician for a rapid and appropriate treatment, particularly in the critical patient.

\section{INTRODUZIONE}

La sepsi è una delle principali cause di morte e con più di 18 milioni di casi anno rappresenta una delle maggiori sfide per la moderna medicina. Il suo numero è andato drammaticamente aumentando con un incremento del 9\% anno negli ultimi 20 anni. In un lavoro del 2005 è riportato che negli Stati Uniti si stimano 750.000 casi ogni anno (7).

Un'indagine del 2003 svolta in Germania evidenzia come vi siano 75.000 casi di sepsi severa all'anno che corrispondono a 110 casi per 100.000 abitanti, poco inferiore all'incidenza dell'infarto miocardico acuto (143 per 100.000). Infatti, con 40.000 morti all'anno, la sepsi severa è la terza causa di morte più frequente in Germania dopo le patologie coronariche (3). In Italia diversi studi hanno messo in evidenza una mortalità del $30 \%$ per la sepsi propriamente detta, del $50 \%$ per la sepsi grave e del 60\% per i casi di MODS (Multi Organ Disfunction Syndrome) $(1,8)$.

Analisi multivariate hanno indicato che un'inadeguata terapia antibiotica è un importante fattore prognostico negativo nella sepsi. È stato dimostrato che, rispetto ad una mortalità media a 30 giorni del $14.7 \%$ nei pazienti con febbre settica, la stessa percentuale scendeva all' $11.8 \%$ in quelli con terapia antibiotica empirica ma adeguata, mentre saliva al $20.1 \%$ in quelli con trattamento inadeguato. L'aumento di mortalità associata a terapia inadeguata diventava ancora più marcato quando venivano chiamati in causa alcuni microrganismi come ad esempio Staphylococcus aureus e Pseudomonas aeruginosa $(6,8,10)$. Le linee guida prevedono che, in carenza di dati microbiologici, si attui una terapia antibiotica empirica con scelta di molecole antibiotiche a largo spettro. Con la disponibilità dei primi dati microbiologici è possibile meglio individuare la terapia e spesso effettuare la cosiddetta "de-escalation" che permette di meglio prevenire l’insorgenza di ceppi resistenti oltre a consentire un significativo risparmio economico. Si capisce pertanto l'importanza di poter disporre in tempi brevi di informazioni microbiologiche clinicamente utili. I metodi tradizionali tuttavia richiedono tempi lunghi, infatti, dal momento dell'avvenuta positività dell' emocoltura, di norma dopo 12-20 ore dal prelievo, si deve effettuare una semina su piastra per ottenere colonie batteriche pure, (ulteriori 18-24 ore) e, successivamente, dopo altre 18-24 ore, si disporrà dei saggi di sensibilità e dell'identificazione definitiva. Il tutto porta ad un referto che risulta disponibile non prima di 48-72 ore $(2,9,12)$.

La possibilità di conoscere in tempi brevi l'antibiotico-sensibilità o resistenza di microrganismi responsabili di sepsi rappresenta quindi un traguardo importante verso una terapia antibio- tica da subito corretta. Considerando che l'utilizzo dello strumento HB\&L ${ }^{\circledR}$ permette di evidenziare la crescita microbica e la sensibilità nei confronti di alcuni antibiotici nell'arco di poche ore, come già precedentemente dimostrato $(4,11)$, abbiamo voluto valutare il suo utilizzo direttamente da emocoltura positiva per saggiare la possibilità di ottenere informazioni clinicamente utili in tempi molto più brevi (5).

\section{MATERIALI E METODI}

Sono stati raccolti $N^{\circ} 91$ ceppi batterici Gram positivi e $N^{\circ} 66$ Gram negativi isolati da emocolture nel periodo dal 1 aprile 2009 al 31 dicembre 2009. Nel dettaglio i microrganismi Gram positivi erano: S. epidermidis (37), S. aureus (17), S. hominis (12), E. faecium (6), S. haemoliticus (5), S. capitis (4), E. faecalis (3), S. lugdunensis (2), E. gallinarum (1), Leuconostoc spp. (1), S. cohnii (1), S. mitis (1), S. saprophyticus (1). I batteri Gram negativi erano: $E$. coli (22), P. aeruginosa (15), $K$. oxytoca (6), E. cloacae (6), K. pneumoniae (5), P. mirabilis (3), A. baumannii (2), E. aerogenes (2), A. ursingii (1), E. agglomerans (1), P. agglomerans (1), S. maltophilia (1), Salmonella gruppo $C$ (1).

Tali ceppi, identificati secondo le normali procedure del nostro Laboratorio, che comprendono Vitek $^{\circledR} 2$ e sistema API ${ }^{\circledR}$ (bioMérieux), sono stati testati per la suscettibilità agli antibiotici utilizzando tre differenti metodologie: Vitek ${ }^{\circledR}$ (bioMérieux), HB\&L ${ }^{\circledR}$ (ALIFAX) ed Etest ${ }^{\circledR}$ (bioMérieux). Facendo riferimento a valori di MIC espressi da CLSI 2009 è stato considerato Etest ${ }^{\circledR}$ come metodica di riferimento.

Gli antibiotici scelti per i saggi di sensibilità sono stati, per i batteri Gram positivi: Vancomicina (VAN), Teicoplanina (TEI), Cefoxitina (CEF); per i batteri Gram negativi: Piperacillina-Tazobactam (PIP/TAZ), Levofloxacina (LVX) ed Amikacina (AMK), per un totale di 471 saggi per ogni metodo analizzato.

Per definire nelle emocolture positive il tipo di microrganismo presente è stata utilizzata la colorazione di Gram eseguita su sedimento centrifugato dell'emocoltura.

Sono state processate unicamente emocolture monomicrobiche.

\section{Test di antibiotico sensibilità con Etest ${ }^{\text {}}$}

Etest ${ }^{\boxplus}$ è stato eseguito seguendo le indicazioni della ditta produttrice direttamente dal brodo dell'emocoltura positiva mediante semina di una goccia di liquido su terreno colturale. I terreni di coltura utilizzati differivano a seconda della morfologia del microrganismo osservato al Gram: cocchi Gram positivi non disposti a grappolo venivano seminati su Mueller

\section{Corresponding author: Vesselina Kroumova}

Laboratorio di Microbiologia e Virologia Azienda Ospedaliera Universitaria "Maggiore della Carità"

28I00 Novara - Corso Mazzini, I8 - Tel. 032 I/373338I - Cell. 335/I90623I - Fax 032I/3733588

E-mail: vesselina.kroumova@maggioreosp.novara.it 
Hinton Agar + 5\% sangue di montone mentre i cocchi Gram positivi disposti a grappolo e i bastoncini Gram negativi erano seminati su Mueller Hinton Agar (BD).

La lettura dei risultati è stata eseguita dopo 18-24 ore seguendo le indicazioni della ditta produttrice e utilizzando l'interpretazione CLSI 2009 (13).

Test di identificazione e antibiotico-sensibilità con Vitek ${ }^{\circledR} \mathbf{2}$ L'emocoltura positiva è stata dapprima seminata su terreno solido Columbia Agar + 5\% sangue di montone e Agar Cioccolato (BD). Il giorno successivo le colonie isolate sono state saggiate per i test di identificazione e antibiotico-sensibilità con le seguenti card: GN e AST-GN27 (bioMérieux) rispettivamente per identificazione e antibiogramma per i microrganismi Gram negativi; GP e AST-P580 rispettivamente per identificazione e antibiogramma per i microrganismi Gram positivi, per l'antibiogramma di enterococchi è stata usata la card AST-P586. Lettura e identificazione sono state eseguite nella giornata successiva tramite lo strumento Vitek ${ }^{\circledR} 2$ seguendo le indicazioni della ditta produttrice.

\section{Esecuzione del test di sensibilità mediante $H B \& L^{\infty}$}

Il sistema $H B \& L^{\circledast}$ utilizza la nefelometria laser (Light Scattering) e consente di monitorare la crescita microbica fornendo una quantificazione della carica batterica in CFU/ml. Le vials dedicate contenenti terreno liquido e sistema di agitazione, vengono inoculate con il campione ed incubate a $37^{\circ} \mathrm{C}$ nello strumento. La presenza di microrganismi causa la deviazione della luce, che viene rilevata da sensori. Il segnale luminoso viene processato da un software che monitorizza le curve di crescita calcolando la carica batterica iniziale.

La metodica da noi seguita per le emocolture positive è stata la seguente: sono stati prelevati $2 \mathrm{ml}$ di sospensione batterica e centrifugati per 10 minuti a $2500 \mathrm{rpm}$. Sono stati inoculati $500 \mu \mathrm{l}$ dello strato sovrapellet in una vial di BHI e successivamente incubati nello strumento $\mathrm{HB} \& \mathrm{~L}^{\circledast}$ a $37^{\circ} \mathrm{C}$. La crescita è stata monitorata e quando la stessa ha raggiunto la concentrazione di 0.5 Mc Farland sono stati allestiti i test di antibiotico-sensibilità. Nel frattempo il sedimento delle emocolture utilizzate per la semina nelle vials HB\&L ${ }^{\circledR}$ è stato osservato al Gram in modo da allestire test di sensibilità in funzio- ne dei microrganismi isolati.

Sono stati inoculati $100 \mu \mathrm{l}$ della sospensione batterica $0.5 \mathrm{Mc}$ Farland nelle vials contenenti brodo eugonico URO-QUICK ${ }^{\circledast}$ KB. Nelle stesse, contestualmente, sono state aggiunte le sospensioni di antibiotico tali che la loro concentrazione fosse equivalente a quella indicata da CLSI per i Break Point dei singoli antibiotici seguendo le indicazioni della ditta produttrice. E stata inoltre seminata un'ulteriore vial per la valutazione della capacità di crescita microbica del campione (curva di riferimento). Una volta avviata l'analisi, grazie al sistema di lettura Light Scattering, si sono ottenute nell'arco di 180 minuti le curve di crescita/inibizione, che consentono di evidenziare la sensibilità o la resistenza del microrganismo nei confronti del singolo antibiotico.

E.coli ATCC 25922, P.aeruginosa ATCC 27853, S.aureus ATCC 25923, ed E. faecalis ATCC 29212, sono stati aggiunti nei saggi di sensibilità come controllo di qualità.

\section{RISULTATI E DISCUSSIONE}

Sono stati analizzati $\mathrm{N}^{\circ} 91$ microrganismi Gram positivi e $\mathrm{N}^{\circ} 66$ Gram negativi. In tutti è stata valutata la loro antibiotico-sensibilità nei confronti delle seguenti molecole: Piperacillina/Tazobactam, Amikacina e Levofloxacina per i batteri Gram negativi; Vancomicina, Teicoplanina e Cefoxitina per i batteri Gram positivi. I dati complessivi sono riassunti nelle Tabelle 1 e 2 dove sono indicati il numero, il tipo e la percentuale di concordanza ottenuti dal metodo HB\&L ${ }^{\circledast}$ vs. Etest ${ }^{\circledast}$ e dal metodo Vitek ${ }^{\circledR} 2$ vs. Etest ${ }^{\circledR}$.

I microrganismi saggiati rappresentano la totalità dei batteri isolati da emocolture monomicrobiche nel periodo preso in esame. In particolare, non essendo scopo della ricerca differenziare fra batteriemie e microrganismi contaminanti, sono stati presi in esame tutti i ceppi di Stafilococchi Coagulasi Negativi (S.C.N.) senza una valutazione della loro rilevanza clinica.

Per ciò che riguarda i saggi relativi alla Cefoxitina si è ben consapevoli delle perplessità relative al suo valore predittivo per l'identificazione di S.C.N. Meticillino Resistenti. Tuttavia lo scopo dell'indagine, come per gli altri antibiotici, è stato unicamente la valutazione di eventuali differenze fra i metodi saggiati.

I risultati ottenuti mostrano una più che buona corrispondenza per entrambi i metodi (vedi Tabella 1).

Tabella I. Percentuali di concordanza totale e discordanza dei singoli antibiotici con i due metodi in esame dei 66 ceppi Gram negativi esaminati.

\begin{tabular}{|c|c|c|c|c|c|c|c|c|c|}
\hline \multicolumn{10}{|c|}{ GRAM NEGATIVI } \\
\hline & ATB & $\mathbf{N}^{\circ}$ Errori & $\%$ tot & Very Major & $\% \mathbf{V M}$ & Major & $\%$ MA & Minor & \% MI \\
\hline \multirow{5}{*}{ HB\&L $^{\circledast}$ vs. ETEST ${ }^{\circledast}$} & PIP/TAZ & 6 & 9,1 & $\mathrm{I}$ & 1,5 & 5 & 7,6 & 0 & 0,0 \\
\hline & $\overline{L V X}$ & 3 & 4,5 & $T$ & 1,5 & 2 & 3,0 & 0 & 0,0 \\
\hline & AMK & 2 & 3,0 & 0 & 0,0 & I & 1,5 & I & $\mathrm{I}, 5$ \\
\hline & TOT & II & 16,7 & 2 & 3,0 & 8 & $12, I$ & I & $\mathrm{I}, 5$ \\
\hline & \% CONC. & 83,3 & & & & & & & \\
\hline \multirow[t]{5}{*}{ VITEK $^{\circledR}$ vs. ETEST ${ }^{\circledR}$} & PIP/TAZ & 8 & $12, \mid$ & 4 & 6,1 & 3 & 4,5 & $\mathrm{I}$ & 1,5 \\
\hline & $\overline{L V X}$ & 0 & 0,0 & 0 & 0,0 & 0 & 0,0 & 0 & 0,0 \\
\hline & $\overline{\mathrm{AMK}}$ & 5 & 7,5 & $\mathrm{I}$ & 1,5 & 3 & 4,5 & 1 & 1,5 \\
\hline & TOT & 13 & 19,6 & 5 & 7,6 & 6 & 9,09 & 2 & 3,0 \\
\hline & \% CONC. & 80,4 & & & & & & & \\
\hline
\end{tabular}

Tabella 2. Percentuali di concordanza totale e discordanza dei singoli antibiotici con i due metodi in esame dei 9 I ceppi Gram-positivi esaminati.

\begin{tabular}{|c|c|c|c|c|c|c|c|c|c|}
\hline \multicolumn{10}{|c|}{ GRAM POSITIVI } \\
\hline & ATB & $\mathbf{N}^{\circ}$ Errori & $\%$ tot & Very Major & $\% \mathbf{V M}$ & Major & $\%$ MA & Minor & $\% \mathrm{MI}$ \\
\hline \multirow[t]{5}{*}{ 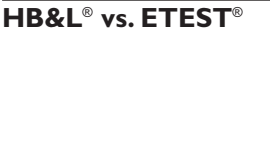 } & VAN & 0 & 0,0 & 0 & 0 & 0 & 0,0 & 0 & 0,0 \\
\hline & TEI & 4 & 4,4 & 0 & 0 & 4 & 4,4 & 0 & 0,0 \\
\hline & CEF & 7 & 7,7 & 2 & 2,2 & 5 & 5,5 & 0 & 0,0 \\
\hline & TOT. & 11 & 12,1 & 2 & 2,2 & 9 & 9,9 & 0 & 0,0 \\
\hline & \% CONC. & 87,9 & & & & & & & \\
\hline \multirow[t]{5}{*}{ VITEK $^{\circledR} 2$ vs. ETEST ${ }^{\circledR}$} & VAN & 5 & 5,5 & 0 & 0,0 & & 0,0 & 5 & 5,5 \\
\hline & TEI & 10 & 11,0 & 0 & 0,0 & 3 & 3,3 & 7 & 7,7 \\
\hline & CEF & 3 & 3,3 & 3 & 3,3 & 0 & 0,0 & 0 & 0,0 \\
\hline & TOT. & 18 & 19,8 & 3 & 3,3 & 3 & 3,3 & 12 & 13,2 \\
\hline & $\%$ CONC & 80,2 & & & & & & & \\
\hline
\end{tabular}

MA, errore maggiore; $\mathrm{Ml}$, errore minore; vedi testo per le abbreviazioni degli antibiotici. 
Sul totale dei 471 test eseguiti ne sono risultati concordanti 449 (95,3\%) con la metodi HB\&L ${ }^{\circledR}$ e 440 (93.4\%) con quella Vitek ${ }^{\circledR}$ 2. Risultati più che soddisfacenti in particolare per $H B \& L^{\circledR}$ vista la rapidità della risposta che questa metodica consente. Sempre in generale si deve rilevare che la maggior parte delle discrepanze di Vitek ${ }^{\circledR}$, in particolare per i Gram positivi, si sono rivelate essere Minor Error.

Più nel dettaglio, per ciò che riguarda le incongruenze riscontrate nei saggi di Teicoplanina fra HB\&L ${ }^{\circledR}$ ed Etest ${ }^{\circledR}$, le risposte di resistenza vs. sensibilità evidenziate si sono verificate sempre, tranne in un caso, nei confronti di ceppi che mostravano una MIC $>=6 \mu \mathrm{g} / \mathrm{ml}$, ciò indica, probabilmente, la necessità di una concentrazione di antibiotico lievemente più elevata nelle vials di $\mathrm{HB} \& \mathrm{~L}^{\circledast}$ onde meglio evidenziare l'attività dell'antibiotico in questione nei confronti di tali ceppi.

I dati relativi alla Vancomicina, per HB\&L ${ }^{\circledast}$, pur molto soddisfacenti, necessitano di ulteriori approfondimenti, in particolare sarà utile saggiare ceppi a sensibilità intermedia per meglio comprendere la capacità di questa metodica di rilevare tali batteri.

Le risposte nei confronti di Piperacillina/Tazobactam, sempre da parte di $\mathrm{HB}^{\mathrm{L}} \mathrm{L}^{\circledR}$, hanno evidenziato che un'osservazione più prolungata (4-6 ore) era in grado di rilevare in 3 dei 5 Major Error, un decremento della curva di crescita dopo la prima fase di innalzamento. Molto probabilmente il fenomeno è da ascrivere all'azione dell'antibiotico sulla parete cellulare. Infatti, in una prima fase pur non realizzandosi la lisi del corpo cellulare l'antibiotico non consente una corretta fase di duplicazione del batterio. Ciò porta alla formazione di lunghi filamenti che determinano un aumento della deviazione della luce. In un secondo tempo l'azione dell'antibiotico porta alla lisi del batterio e conseguentemente alla caduta della curva di crescita. Questi ceppi devono quindi essere correttamente considerati come sensibili.

Per ciò che riguarda Amikacina, sempre per la metodica $H B \& L^{\circledast}$, in alcuni casi è stata osservata una curva di crescita che, seppur molto più lentamente rispetto alla curva di riferimento, evidenziava una chiara crescita batterica. Il software dello strumento, utilizzando propri algoritmi, pur con percentuali variabili di sensibilità, interpretava il ceppo in questione come sensibile. Tale interpretazione era confermata sia dai risultati ottenuti con Etest ${ }^{\circledR}$ che da quelli con Vitek ${ }^{\circledR}$. Un tale fenomeno richiede, tuttavia, un ulteriore approfondimento per meglio comprendere le cause di questo comportamento e valutarne le eventuali ricadute cliniche.

Altro dato di notevole interesse è il tempo medio impiegato dai vari ceppi batterici a raggiungere la concentrazione di $0.5 \mathrm{Mc}$ Farland. L'analisi dei tempi di crescita ha mostrato che per i batteri Gram positivi questo è stato in media di 174 minuti (range 85-345). Mentre per i microrganismi Gram negativi il tempo medio è stato di 114 minuti (range 40-320). Tali tempi confermano che nella maggioranza dei casi, specie per batteri Gram negativi, è possibile giungere ad un risultato, seppur preliminare, di antibiotico-sensibilità o resistenza in meno di sei ore dalla positivizzazione dell'emocoltura.

In conclusione valutando nel loro complesso i dati ottenuti con la metodica HB\&L ${ }^{\circledast}$ si evidenzia come su 471 saggi solo in 4 casi si siano riscontrati Very Major Error con una discordanza dello $0.9 \%$. È rilevante sottolineare che l'identificazione di una falsa sensibilità ad un dato antibiotico risulta quindi evento del tutto raro. Inoltre bisogna considerare che il risultato ottenuto con la nostra metodica è un dato preliminare che dovrà poi essere confermato da test ufficialmente riconosciuti. Si può ancora aggiungere che spesso le curve di crescita, in particolare dopo una prima fase di utilizzo dello strumento ed una conseguente maggior conoscenza delle loro caratteristiche, consentono di intravedere quale dei tre antibiotici saggiati possa mostrare una miglior attività verso il ceppo batterico in esame. Per tutti questi motivi si può tranquillamente ritenere che la metodica da noi approntata è in grado di fornire importanti indicazioni cliniche in tempi ridottissimi, cosa che risulta di rilevante importanza anche alla luce della gravità dell'evoluzione della sepsi nei casi in cui non viene instaurata da subito una corretta terapia antibiotica empirica (8).

In definitiva questa metodica, pur necessitando ancora di una più completa standardizzazione, è in grado di offrire, nelle mani di uno specialista microbiologo, significativi vantaggi al clinico grazie alla rapidità con la quale vengono resi disponibili i dati di sensibilità o resistenza mantenendo nel contempo una riproducibilità degli stessi analoga ad uno dei più diffusi test in commercio.

\section{RINGRAZIAMENTI}

Gli autori ringraziano la Ditta ALIFAX per il sostegno dato a questo studio

\section{BIBLIOGRAFIA}

1. Bienza N, Fumagalli R, Antonelli M, Langer M, Gruppo italiano replay. Epidemiologia della sepsi in Italia. Minerva Anestesiologica, 2005; 71 (Suppl. 1 al n¹0): 364-6.

2. Bloos F, Hinder F, Becker K, et al. A multicenter trial to compare blood culture with polymerase chain reaction in severe human sepsis. Intensive Care Medicine, 2010; 36(2): 241-7.

3. Brunkhorst FM, Engel C, Reinhart K, et al. Epidemiology of severe sepsis and septic shock in Germany - results from the German prevalence study. Crit Care 2005; 9 (suppl.1): 196.

4. Cagnacci S, Cavallini F, Maioli E, et al. Utilizzo del sistema Uro-Quick per l'identificazione rapida di batteri produttori di ?-lattamasi a spettro esteso (ESBL). Microbiologia Medica, 2005; 20(2): 68-70.

5. Fontana C, Favaro M, Minelli S, Bossa MC, Altieri A, Favalli C. A nove culturing system for fluid samples. Med Sci Monit, 2009; 15(2): 55-60.

6. Fraser A, Paul M, Almanasreh N, et al. Benefit of appropriate empirical antibiotic treatment: thirty-day mortality and duration of hospital stay. The American Journal of Medicine, 2006; 119 (11): 970-6.

7. Konrad R. Il problema Sepsi. Expert Symposium - PCR-based identification of bloodstreem infections - Berlino, 23 giugno 2005.

8. Kumar A, Roberts D, Wood KE, et al. Duration of Hypotension before initiation of effective antimicrobial therapy is the critical determinant of survival in human septic shock. Critical Care Medicine, 2006; 34:1589-96.

9. Levi MM, Dellinger RP, Towsend SR, et al. The surviving sepsis compaign: result of an international guideline-based performance improvement program tergeting severe sepsis. Intensive Care Medicine, 2010; 36: 222-31.

10. MacArthur RD, Miller M, Albertson T, et al. Adequacy of early empiric antibiotic treatment and survival in severe sepsis: experience from the MONARCS trial. Clinical Infectious Diseases, 2004; 38: 284-8.

11. Roveta S, Cagnacci S, Cassanelli C, Cavallini F, Debbia EA, Marchese A. Utilizzo del sistema Uro-Quick in procedure rapide di screening per la determinazione della sensibilità agli antibiotici in alcuni important microrganismi patogeni. GIMMOC, 2006; 10 (1): 11-20.

12. Struelens MJ. Detection of microbial DNAemia: does it matter for sepsis management? Intensive Care Medicine, 2010; 36: 193-5.

13. Wikler MA, Cockerill FR, Bush K, et al. Methods for dilution antimicrobial susceptibility tests for bacteria that grow aerobically; approved standards-eight edition. Clinical and Laboratory Standards Institute (CLSI) 2009; 29 (2). 